\title{
Dysmorphophobic Patient Seeking Primary Cosmetic Rhinoplasty ${ }^{*}$
}

\author{
Hashem Shemshadi \\ Plastic and Reconstructive Surgery, University of Social Welfare and Rehabilitation Sciences, \\ Tehran, Iran \\ Email: shemshadii@gmail.com, shemshadi@uswr.ac.ir
}

Received November 25, 2012; revised March 2, 2013; accepted April 15, 2013

Copyright (C) 2013 Hashem Shemshadi. This is an open access article distributed under the Creative Commons Attribution License, which permits unrestricted use, distribution, and reproduction in any medium, provided the original work is properly cited.

\begin{abstract}
This article is aimed to elaborate the significance of detecting clinical features (subjectivity, objectivity and assessment) of the Dysmorphophobic (DMP) psychiatric patients seeking primary cosmetic rhinoplsty (PCR). DMP clients present as a fixation of their thoughts toward the trivial flaws on their body's anatomy. They consider such minor defects as major and show a great amount of anxiety for such negligible issues. Such dread affects their social, occupational and family's life and trigger them to seek means of correcting such small blemishes through medical and or surgical approaches. Considering to their nose, they crave to eliminate their minor defects through PCR operation. PCR is viewed as one of the most prevalent aesthetic operations in the field of cosmetic surgery. Author's method of approach in PCR is to select candidates after obtaining a meticulous health history, physical exam and appropriate para clinical tests. In some uncertained cases, based on their subjectivity and objectivity presentations, are referred for consultation with a psychiatrist for final assessment. Most selected patients undergo PCR via standby anesthesia through an incision on the nasal columella (open rhinoplasty), or in others which their nasal tip alternation is not needed, PCR is approached with no incision on the nasal columella (closed rhinoplasty). Due to the high demand of PCR in the world, among many clients who are seeking for such surgery, might be some cases with DMP disorder who are overlooked in spite of an accurate surgeon's screening.
\end{abstract}

Keywords: Rhinoplasty; Dysmorphophobia; Cosmetic Surgery; Psychiatric Consultation

\section{Introduction}

Desiring to "look younger" is the essential nature of all human beings. People try to appear attractive, impressive and younger in their social life. Success in life, truly has been partially correlated to such values, especially if such qualities link with the business competitions [1].

In most instances, people are willing to undergo some kind of procedures which make them look younger, acceptable and appealing [2]. If such aspirations remain within a normal wish and logic, is fine, but if such inclinations go beyond realities, would create problems rather than benefits [3]. Prospects beyond actualities, create emotional and psychological harm if are not obtained [4]. These emotional and psychological deteriorations not only affect the patients' own quality of life but also their loved ones as well [5]. Since PCR is considered as one of the common nasal aesthetic procedure, is also one of the

\footnotetext{
${ }^{*}$ This review article is prepared with no conflict of interest.
}

common procedures which patients with DMP disorder who are so thoughtful about their trivial nasal defects, are hunting for.

Even in non DMP cases, that the patients' expectation which is not obtained will cause deterioration of their self esteem and self confidence in post operative periods [6]. These mentioned problems result even more serious snags in patients with the DMP psychiatric disorder [7]. In their initial interview, DMP cases discuss issues which are not accessible and if they undergo PCR by a wrong selection, will remain dissatisfied, no matter how reasonable would be the PCR surgical results [8].

\section{DMP Patients and PCR Surgeons}

Patients with DMP disorder, show restlessness, anxiety and hopelessness in their first office visit. Most of them have no logic behind their decision and plannings toward PCR operation. Such DMP manifestation may be the sign and symptoms of other more serious underlying psycho- 
logical ailments such as major depression and or schizophrenia [9]. In rare situations such mentally disturbed individuals may end up into suicidal attempts [10]. Non noticeable remarks and minor complication such as post operative olfactory malfunctioning, may look so severe and crippling [11]. They get so emotionally superficial, laugh easily and burst to cry simply if someone addresses their PCR after surgery. If some other major complications occur, it will be really difficult and mostly impossible to handle in such DMP patients [12].

As cosmetic surgeons become more experienced, they learn many lessons which are not written in the aesthetic surgical texts and or they have not been faced during their cosmetic surgical trainings [13]. As they practice more, they may easily differentiate the DMP patients seeking for PCR, versus those who are possessing a reasonable mental health for choosing PCR. In cases of uncertainty, a clever cosmetic surgeon, asks consultation with a psychiatrist [14]. In rare situations such DMP patients are failed to be detected by the cosmetic surgeons. In this situation, unpleasant office visits begin and inconveniences are created for the DMP patient and the cosmetic surgeon both. Sometimes such aforementioned problems diminish with time, but in some other circumstances nuisance remain long and patients may need more serious psychiatric cares [15].

\section{Screening DMP Patients}

One of the sign of progress for any PCR surgeon is to screen who is a proper candidate for PCR operation and who is not. Appropriate candidate shows a calm personality along with conversing based on facts. They show understanding and a feel of accepting any side effects that may possibly arise. Usually they have previously seen your similar PCR works and have been oriented all the likely happenings which may happen after surgery. Author considers all of the aforementioned criteria in deciding to choose a patient to undergo PCR. As mentioned earlier, as the time passes and the cosmetic surgeons operate many patients, they learn so many clues which are not written in the texts. As their practice progresses, they may have encountered many psychologically disturbed patients who have been seeking PCR surgery. They may have also missed some others who have had the DMP individuals who have undergone PCR. Some of these cases may show an emotional and psychological reaction in asking the PCR surgeons to accept them for rhinoplasty surgeries [16].

Psychological screening of most DMP patients may be applied easily with no complexity, but in some other cases such selection may remain obscure. In some occasions, patients' external motivations for PCR are logical, but in some others, inspirations may be idealistic and non accessible [17]. Screening of the patients who seek PCR and have been labelled as possible DMP is highly recommended to be consulted by a psychiatrist for final assessments. This consultation proceeds PCR surgeons to an early detection of DMP patients and be aware of their real reasoning behind their PCR decision [18].

\section{Author's Approach}

The author's approach in selecting of cases for PCR are based on a complete consideration on their past health histories and results of my general and specifically nasal physical exams. I also observe their mode of interactions in clinical setting. I do listen carefully to their words of expectations. Prior to surgery, informed consents are read, understood and signed by the case with a witness. Patients who are exaggerating on my reputation and think they would impress me can convince me to meet their expectations in PCR, are considered as a red flag sign to be chosen. Patients who desire to look like a celebrity's photo must be reconsidering their selection and most of all, if they complain about very trivial defects and desire to be eliminated perfectly, also is a red flag sign. These matters may show a direct and or an indirect sign of DMP disorder. In these circumstances, I will consider more visiting and or request needy laboratory tests along with referring them for a psychiatric consultation.

In spite of such care taken and being exact in selection for PCR, there are some patients who insisting to undergo such operation by the cosmetic surgeon and I have frequently faced with such clients in my more than two decades of PCR experiences. In such situation, the surgeon may be under the influence of patient's emotional and psychological reactions of being accepted for PCR [19]. In such situation surgeon may feel under pressure to say "yes" or "no" to such candidates [20]. Patients may even deny any psychiatric disorders and show their reasoning for PCR completely logical [21]. Persistency is a hallmark of more probable diagnosis of DMP in such cases and should be given a second thought for selection for PCR [22]. In such stipulation, I am usually firm in saying "no" and not selecting such candidate for a PCR operation [23].

Selecting such possible DMP patients would create an unpredictable outcome. Their mental disorder would alter an absolutely satisfactory result into a totally dissatisfied outcome. Their mind is occupied in post operatively with thinking the minute imperfections in their nose and continuously asking for correcting such trivial defects [24]. In these conditions, I usually try to revisit such patients more frequent, discussing issues clearly with them and their loved ones and finally asking for the psychiatric assists for possible antipsychotic medical therapies.

As time passes by, I am being so inspective in my clients selection for PCR. I believe, if the initial selection takes place correctly, most patients feel good about the 
results of their cosmetic nasal surgery and express their satisfactions for the PCR consequence [25].

\section{DMP Patient in Pre and Post PCR}

Routinely, final decision made after discussing the main issues of the PCR operation with all patients. They should be well informed about the costs, type of anesthesia, hospital stay duration and post surgical cares. A next clinic appointment is set after surgery for checking the patient's condition and their possible dressings change.

DMP individual who has been selected for PCR by mistake, usually shows low interest towards such office's clinical visits and regulations and usually exhibit disinterest in keeping their appointments [26,27]. They bringup new issues about their nose and express their dissatisfactions for the PCR results .They usually exhibit low collaboration and poor communication with their cosmetic surgeon [28]. In most situations, they show rigidity and express pessimistic views in their office visits.They also seek other cosmetic surgeons for their possible nasal re operation [29]. They may have a great tendency for undergoing a second, third and even more nasal re-operations by other cosmetic surgeons [30].

\section{Final Words}

Precise evaluations in patients who refer for the PCR, by complete observing their clinical visits and accurately listening to their words of expectations in relation to their nasal anatomy defect, cosmetic surgeons most likely can note the unsure character of clients with DMP psychotic disorder. Final planning for such operations is relied upon a psychiatric consultation which clears most obscures and enables cosmetic surgeons to select the right patients for PCR operations.

\section{REFERENCES}

[1] O. Babuccu, et al., "Sociological Aspects of Rhinoplasty,” Aesthetic Plastic Surgery, Vol. 27, No. 1, 2003, pp. 44-49. doi:10.1007/s00266-002-1517-9

[2] S. E. Moolenburgh, M. A. Mureau and S. O. Hofer, "Facial Attractiveness and Abnormality of Nasal Reconstruction Patients and Controls Assessed by Laypersons," Journal of Plastic, Reconstructive \& Aesthetic Surgery, Vol. 61, No. 6, 2008, pp. 676-680. doi:10.1016/j.bjps.2007.12.017

[3] R. L. Kurtzberg, et al., "Psychologic Screening of Inmates Requesting Cosmetic Operations: A Preliminary Report," Plastic and Reconstructive Surgery, Vol. 39, No. 4, 1967, pp. 387-396. doi:10.1097/00006534-196704000-00009

[4] R. Zojaji, et al., "High Prevalence of Personality Abnormalities in Patients Seeking Rhinoplasty,” Otolaryngology-Head and Neck Surgery, Vol. 137, No. 1, 2007, pp. 83-87. doi:10.1016/j.otohns.2007.02.027
[5] S. A. Yellin, "Aesthetics for the Next Millennium," Facial Plastic Surgery, Vol. 13, No. 4, 1997, pp. 231-239. doi:10.1055/s-0028-1082423

[6] S. Ghadakzadeh, et al., "Body Image Concern Inventory (BICI) for Identifying Patients with BDD Seeking Rhinoplasty: Using a Persian (Farsi) Version,” Aesthetic Plastic Surgery, Vol. 35, No. 6, 2011, pp. 989-994. doi:10.1007/s00266-011-9718-8

[7] D. Veale, L. De Haro and C. Lambrou, "Cosmetic Rhinoplasty in Body Dysmorphic Disorder,” British Journal of Plastic Surgery, Vol. 56, No. 6, 2003, pp. 546-551. doi:10.1016/S0007-1226(03)00209-1

[8] K. Yu, A. Kim and S. J. Pearlman, "Functional and Aesthetic Concerns of Patients Seeking Revision Rhinoplasty," Archives of Facial Plastic Surgery, Vol. 12, No. 5, 2010, pp. 291-297. doi:10.1001/archfacial.2010.62

[9] M. Gipson and F. H. Connolly, "The Incidence of Schizophrenia and Severe Psychological Disorders in Patients 10 Years after Cosmetic Rhinoplasty," British Journal of Plastic Surgery, Vol. 28, No. 3, 1975, pp. 155-159. doi:10.1016/0007-1226(75)90119-8

[10] C. S. Thomas and D. P. Goldberg, "Appearance, Body Image and Distress in Facial Dysmorphophobia," Acta Psychiatrica Scandinavica, Vol. 92, No. 3, 1995, pp. 231236. doi:10.1111/j.1600-0447.1995.tb09574.X

[11] H. Shemshadi, et al., "Olfactory Function Following Open Rhinoplasty: A 6-Month Follow-Up Study,” BMC Ear, Nose and Throat Disorders, Vol. 8, 2008, p. 6. doi:10.1186/1472-6815-8-6

[12] J. Wind, "Blindness as a Complication of Rhinoplasty," Archives of Otolaryngology_Head and Neck Surgery, Vol. 114, No. 5, 1988, p. 581. doi:10.1001/archotol.1988.01860170111036

[13] H. J. Thakar, P. E. Pepe and R. J. Rohrich, "The Role of the Plastic Surgeon in Disaster Relief," Plastic and Reconstructive Surgery, Vol. 124, No. 3, 2009, pp. 975-981. doi:10.1097/PRS.0b013e3181b17a7a

[14] H. S. Thomson, "Preoperative Selection and Counseling of Patients for Rhinoplasty," Plastic and Reconstructive Surgery, Vol. 50, No. 2, 1972, pp. 174-177. doi:10.1097/00006534-197208000-00013

[15] J. R. Anderson and M. Willett, "On Planning before Rhinoplasty,” Laryngoscope, Vol. 94, No. 8, 1984, pp. 1115-1116. doi:10.1288/00005537-198408000-00025

[16] P. Marcus, "Some Preliminary Psychological Observations on Narcissism, the Cosmetic Rhinoplasty Patient and the Plastic Surgeon," Australian and New Zealand Journal of Surgery, Vol. 54, No. 6, 1984, pp. 543-547. doi:10.1111/j.1445-2197.1984.tb05443.x

[17] A. J. Tasman, "The Psychological Aspects of Rhinoplasty," Current Opinion in Otolaryngology \& Head and Neck Surgery, Vol. 18, No. 4, 2010, pp. 290-294. doi:10.1097/MOO.0b013e32833b51e6

[18] J. G. Stafne, “The Cosmetic Surgery Patient. Why Do They Do It to Themselves?” Minnesota Medicine, Vol. 63, No. 3, 1980, pp. 175-177, 209.

[19] L. Meyer and S. Jacobsson, "Psychiatric and Psychosocial Characteristics of Patients Accepted for Rhino- 
plasty,” Annals of Plastic Surgery, Vol. 19, No. 2, 1987, pp. 117-130. doi:10.1097/00000637-198708000-00003

[20] R. J. Rohrich, "Streamlining Cosmetic Surgery Patient Selection-Just Say No!” Plastic and Reconstructive Surgery, Vol. 104, No. 1, 1999, pp. 220-221. doi:10.1097/00006534-199907000-00034

[21] R. Feiss and J. P. Real, "Rhinoplasty: Psychological Aspects. Psychiatrist/Surgeon Collaboration. Apropos of 207 Patients Surgically Treated 1 or More Times between 1980 and 1986," Annales de Chirurgie Plastique et Esthetique, Vol. 34, No. 5, 1989, pp. 392-394.

[22] C. Chalier, "Plastic Surgery. Psychological Aspects," Soins en Chirurgie, No. 64-65, 1986, pp. 27-29.

[23] J. B. Copas and A. A. Robin, "The Facial Appearance Sorting Test (FAST): An Aid to the Selection of Patients for Rhinoplasty,” British Journal of Plastic Surgery, Vol. 42, No. 1, 1989, pp. 65-69.

[24] P. Haraldsson, "Psychosocial Impact of Cosmetic Rhinoplasty,” Aesthetic Plastic Surgery, Vol. 23, No. 3, 1999, pp. 170-174. doi:10.1007/s002669900264

[25] P. B. Dinis, M. Dinis and A. Gomes, "Psychosocial Consequences of Nasal Aesthetic and Functional Surgery: A Controlled Prospective Study in an ENT Setting," Rhi- nology, Vol. 36, No. 1, 1998, pp. 32-36.

[26] D. B. Sarwer, "Discussion: High Prevalence of Body Dysmorphic Disorder Symptoms in Patients Seeking Rhinoplasty,” Plastic and Reconstructive Surgery, Vol. 128, No. 2, 2011, pp. 518-519. doi:10.1097/PRS.0b013e31821e7248

[27] K. A. Phillips, "Psychosis in Body Dysmorphic Disorder,” Journal of Psychiatric Research, Vol. 38, No. 1, 2004, pp. 63-72. doi:10.1016/S0022-3956(03)00098-0

[28] M. Javanbakht, et al., "Body Dysmorphic Factors and Mental Health Problems in People Seeking Rhinoplastic Surgery," Acta Otorhinolaryngologica Italica, Vol. 32, No. 1, 2012, pp. 37-40.

[29] E. F. Williams 3rd and S. M. Lam, "A Systematic, Graduated Approach to Rhinoplasty," Facial Plastic Surgery, Vol. 18, No. 4, 2002, pp. 215-222. doi:10.1055/s-2002-36489

[30] B. T. Ambro and R. J. Wright, "Psychological Considerations in Revision Rhinoplasty," Facial Plastic Surgery, Vol. 24, No. 3, 2008, pp. 288-292. doi:10.1055/s-0028-1083083 